\title{
Explaining the Scientific Method to First Year Biology Students in An Accessible Manner
}

\author{
Charlotte de Araujo, Nicole Pereira, Karen J. Puddephatt, Lynda H. \\ McCarthy, and Andrew E. Laursen
}

\author{
Ryerson University, Department of Chemistry \& Biology, 350 Victoria St, Toronto ON M5B \\ 2K3 CAN \\ (cdearaujo@ryerson.ca; nicole.pereira@ryerson.ca; karenp@ryerson.ca; \\ 13mccart@ryerson.ca; alaursen@ryerson.ca)
}

\begin{abstract}
The scientific method paired with the practice of statistics is widely implemented to analyze, interpret and derive conclusions regarding a dataset. This laboratory exercise introduced first year biology students to the scientific method, applying it to evaluate how brown planaria (Dugesia tigrina) responds to light. In the laboratory setting, students were required to formulate a research question and hypothesis, design a study, record observations, gather data and perform statistical calculations (mean, median, standard deviation, ttests), receiving feedback from their peers and teaching assistants. When surveyed, less than half of the students felt they had a strong background in mathematics or were comfortable in the subject. To resolve this, we produced multiple videos enabling students to review the content to help recall the material. These resources introduced students to terminology, provided them opportunities to practice usage of these terms, as well as instructions on how to perform statistical calculations. We employed an analysis of covariance (ANCOVA) to determine if the students who viewed the videos performed better on the statistical analysis and interpretation portion. Based on ANCOVA, the wet laboratory experiments in conjunction with video resources, resulted in increased student performance in the laboratory component compared to previous iterations without video tools.
\end{abstract}

Keywords: statistics, scientific method, introductory biology

Link to Original Poster File: https://doi.org/10.37590/able.v41.poster55

\section{Introduction}

At Ryerson University, for students enrolled in first year biology (Biology I), the laboratory component consists of five wet laboratories offered in a biweekly mode. We devised the first laboratory to introduce the scientific method and provide fundamental statistics concepts (Laursen et al 2018). Applying statistics to biological research allows biologists to paint a bigger picture on what their findings truly entail and helps them predict outcomes of their treatments. Due to the large class size and time constraints (2.5 hour session) we were interested in additional means to disseminate laboratory components to help reinforce these applied concepts. To help bridge the temporal separation between the lab offerings as well as the lecture material we incorporated supplementary videos.
To determine the need for supplementary tools, such as videos, to students by professors, students from Ryerson University's Faculty of Science first-year general Biology I laboratories were surveyed. Approximately 78\% of first-year science students $(\mathrm{n}=483)$ enrolled in Ryerson University's first-year Biology I (BLG143) felt that it would be beneficial if a professor provided them with more informative tools, such as videos. It was also found that only $24 \%$ of students preferred to use the textbook and class notes to gain knowledge, rather than using an external tool, such as a video. In fact, $79 \%$ of science students were already using video formats to recall knowledge, skills, and to learn more about concepts outside of the classroom. This data supports the high demand for professors to produce or select videos to supplement their lectures. Students enrolled in Biology I are already using videos to teach themselves concepts, and professors need to ensure the videos they are observing are relevant to their material. 
First-year undergraduate students were surveyed to determine if they felt that they had a strong background in mathematics and if they felt comfortable in the subject to assess math anxiety. The theory behind math anxiety is that students who feel anxious regarding math perform worse and this is an extremely common anxiety amongst students globally (Foley et al. 2017). It is very common in STEM related fields given that mathematics is an integral component to it (Foley et al., 2017). Many students struggle with mathematics because it can be a complex subject and as a result, develop an anxiety around the subject (Foley et al., 2017). Only 16\% of BLG143 students felt that they did not have a strong background in mathematics and did not feel comfortable in the subject. The remaining students either felt neutral towards this statement $(35 \%)$ or agreed with it $(47 \%)$.

In this introductory laboratory module, we coupled supplementary video resources with the laboratory material. The topics include: i) Overview of the scientific method (https://youtu.be/J8HM1i_0cIY) ii) Description of t-tests (https://youtu.be/fbeB9h IIN4) and iii) Introduction to basic laboratory statistics (https://www.youtube.com/watch? $\mathrm{v}=\mathrm{mW}$ 5axdfyYhc\&t $=9$ 3s). The composition of the third video was influenced by a study performed by Dr. Stephanie Gray Wilson, 'The
Flipped Class: A Method to Address the Challenges of an Undergraduate Statistic Course'. It started with building a small foundation in the subject (Wilson 2013) by having the importance of statistics in sciences discussed and by having basic terms that will be commonly used in laboratory introduced. The terms defined include: independent variable, dependent variable, control, treatment, quantitative data, qualitative data, population, sample, central tendency (mean, median, and mode), and histogram. Definitions of the key terms presented within the video were available audibly, visually through pictures, and also provided in text form. The video also discussed the application portion of statistics (Wilson 2013), which in the video was the description and example of application for central tendency. This definition was expressed using an example of classroom grade differences. Methods of organizing data were also considered an applied process, specifically understanding histograms in the video. This definition was expressed using an example of how a biologist studies different lengths of snakes. A final summary was created for the ending to review all key terms discussed in video to help reinforce concepts presented in the laboratory. 


\section{Student Outline Introduction to the Scientific Method}

\section{Objectives}

- $\quad$ To use the Scientific Method

- $\quad$ Be able to correctly write a null and alternate hypothesis

\section{Introduction}

Brown planaria (Dugesia tigrina) are free-living flatwoms that are found in many aquatic habitats (note: planarian is the singular form of the noun, planaria is the plural form of the noun). They have become important model systems for studying stem cell biology, regeneration, neurological development, and genetics. They are ecologically important in the processing of detritus (dead organic matter and bacteria) in sediments of lakes and ponds. They are one of the simplest animals that exhibits cephalization and processing of information from sensory organs. In particular, they have simple cerebral eyes that are capable of sensing light and responding. The eyes are on the planarias' dorsal sides. The eyes are composed of two cell types - pigment cells and photoreceptor neurons. The pigmented cells form a semi-lunar optic cup and functions in absorption of light. The orientation of the light cup confers a left-right directional and an anterior-posterior directionality, so the animal is able to detect the direction of the light source. The photoreceptor cells are bipolar neurons, with the cell bodies located within the optic cups. The dendrites of the photoreceptor cells contain opsins, G-protein coupled receptors. Rhodopsin is a complex of the opsin and a chromophore that actually absorbs the light. Different chromophores exist in the animal kingdom and have different optical properties (i.e. absorb different wavelengths of light) as a function of their different molecular structures. There are also a variety of opsins in the animal kingdom that bond with the various chromophores. The result is that different lineages of animals detect different wavelengths of light. As animals that dwell in sediments, and that are eaten by visual predators, how do you expect that planaria will respond to light? Formulate a hypothesis relating to behaviour of planaria in response to light.

\section{Methods and Data Collection}

\section{Part A: Asking Questions}

First, we are going to consider the question of what to expect. Should movement differ under conditions of directional light versus diffuse light? You may have some ideas on what to expect when you consider the ecology of the planaria. For example, if I think that the planaria will avoid light, I might formulate the question: "Do planaria move away from a directional light source?"

Formulate your research question and record it on the Laboratory 1 Report Sheet (Question 5).

\section{Part B: Formulating a Hypothesis}

Based on your research question, you will formulate a null hypothesis and alternate hypothesis that can be used to test whether the stimulus does affect movement of planaria. In formulating null and alternate hypotheses, notice that the null hypothesis is always that there is no difference between your experimental groups, and the alternative hypothesis is that there is a difference.

Based on your own research question (recorded in the Laboratory Report Sheet), formulate a Null Hypothesis and an Alternate Hypothesis. Discuss these with your Teaching Assistant (TA) to be sure they meet the requirements of testable hypotheses. Then record your hypotheses on the Laboratory 1 Report Sheet (Question 6).

\section{Part C: Experimentation and Making Observations}

In this experiment, you are determining how far planaria move in three minutes in response to light (directional versus diffuse). If you have 10 different pairs of lab partners in your section, and each pair of students observes one planarian under diffuse light conditions, and one planarian under directional light conditions, then you will have 10 replicates for each treatment (light condition). Your actual number will vary, depending on the number of student pairs in your lab section.

You will also have a clearly defined independent variable. This is the variable that you think affects movement of the planaria and is the variable which you manipulate. In this example, the independent variable is light conditions (directional versus diffuse). And, you have a clearly defined dependent variable, which is the variable you are measuring. What is the dependent variable in your experiment?

Finally, you have controlled variables. These include temperature, humidity, noise. These are the variables that might affect movement of the planaria, but which you make sure were the same for each experimental group. Therefore, there could be no effect of temperature (for example) on the outcome since it was the same for both groups. 
Data collected in experiments are usually presented visually in the form of tables or graphs. In this case, we will use a table, and one is provided for you in the Laboratory Report Sheet. On the Laboratory Report Sheet, identify your independent variable, your dependent variable, and some of your controlled variables (Question 7).

\section{Procedure}

Each pair of students will receive 2 planaria. Each planarian will be transferred to a rectangular plastic tray (one planarian per tray). The planarian will be placed near one end of each tray. Using a permanent marker, make a mark on the side wall of the tray to indicate where (along the length of the tray) the planarian starts. Do so for each tray.

On your bench, there will be two boxes. One box has a white lid that permits diffuse light from the lab to enter the box. There should be no directionality to this light. Place one of the plastic trays in this box with the white lid covering it. The second box contains a white LED at one end of the box. Place the remaining plastic tray such that the end containing the planarian is just under the LED light source.

Set a timer and permit 3 minutes for your planaria to move.

After 3 minutes, use a permanent marker to mark the location of the planarian on the side wall of the tray. Measure the distance traveled over 3 minutes by each planarian. If the planarian moved toward the far end of the tray, this would be recorded as a positive value. If the planarian moved even closer to the wall where it was initially placed, this would be recorded as a negative value.

Record the distance traveled by each planarian in the table in your Laboratory 1 Report Sheet (Question 8). Then obtain values recorded by all other students in your laboratory section. Your TA will facilitate this by having students record these values on the board at the front of the lab

\section{Part D: Determining Variation Among Your Observations}

You will have two observational (or sample) populations in the data recorded on your table (Question 8). The first population are those planaria that were exposed to diffuse light, and the second population are those planaria that were exposed to directional white light. After you have recorded the distances traveled (including your own planaria and those of your lab mates), you will create a histogram to demonstrate graphically the variation among your sample populations. Suppose that you made the following recordings:

Table 1. Sample recordings.

\begin{tabular}{|c|c|c|c|c|}
\hline $\begin{array}{c}\text { Reference } \\
\text { (diffuse light) }\end{array}$ & $\begin{array}{c}\text { Distance traveled } \\
(\mathrm{mm})\end{array}$ & 2 & $\begin{array}{c}\text { Treatment } \\
\text { (directional light) }\end{array}$ & $\begin{array}{c}\text { Distance traveled } \\
(\mathrm{mm})\end{array}$ \\
\hline 1 & 2 & 1 & 4 \\
\hline 2 & 3 & 2 & 2 \\
\hline 3 & 5 & 3 & 9 \\
\hline 4 & -1 & 4 & 5 \\
\hline 5 & 0 & 5 & 3 \\
\hline 6 & 6 & 6 & 10 \\
\hline 7 & 3 & 7 & 7 \\
\hline 8 & 0 & 8 & 6 \\
\hline 9 & -2 & 9 & 4 \\
\hline 10 & 4 & 10 & 3 \\
\hline
\end{tabular}

The distances traveled cover a range from $-2 \mathrm{~mm}$ to $10 \mathrm{~mm}$. Next, we want to create "bins" that will allow us to lump together all observations that fall within a given range, for each of the two populations. We could have as many as 14 bins (bin $1=-2 \mathrm{~mm}$, bin $2=-1 \mathrm{~mm}$, bin $3=0 \mathrm{~mm}$, etc.), however that would not really be a useful way to aggregate data and get a better sense of overall patterns. A smaller number of evenly sized bins might be better. For example, let's consider 7 bins, where bin $1=-2$ to $-1 \mathrm{~mm}$, bin $2=0$ to $1 \mathrm{~mm}$, etc. (see below). We next record the total number of observations that fit within each bin. For example, bin 1 ( -2 to $-1 \mathrm{~mm}$ ) includes two observations for planaria under reference light conditions (diffuse light) and includes zero observations for planaria under treatment light conditions (directional light). 
Table 2. Sample recordings.

\begin{tabular}{|l|l|l|}
\hline Bins & $\begin{array}{l}\text { Reference (diffuse light) } \\
\text { \# observations }\end{array}$ & $\begin{array}{l}\text { Treatment (directional light) } \\
\text { \# observations }\end{array}$ \\
\hline $1(-2$ to $-1 \mathrm{~mm})$ & 2 & 0 \\
\hline $2(0$ to $1 \mathrm{~mm})$ & 2 & 0 \\
\hline $3(2$ to $3 \mathrm{~mm})$ & 3 & 2 \\
\hline $4(4$ to $5 \mathrm{~mm})$ & 2 & 3 \\
\hline $5(6$ to $7 \mathrm{~mm})$ & 1 & 3 \\
\hline $6(8$ to $9 \mathrm{~mm})$ & 0 & 1 \\
\hline $7(10$ to $11 \mathrm{~mm})$ & 0 & 1 \\
\hline
\end{tabular}

Next, we plot our histogram (Fig. 1.3), which has frequency (or number of observations) on the y-axis and bin sizes on the x-axis:

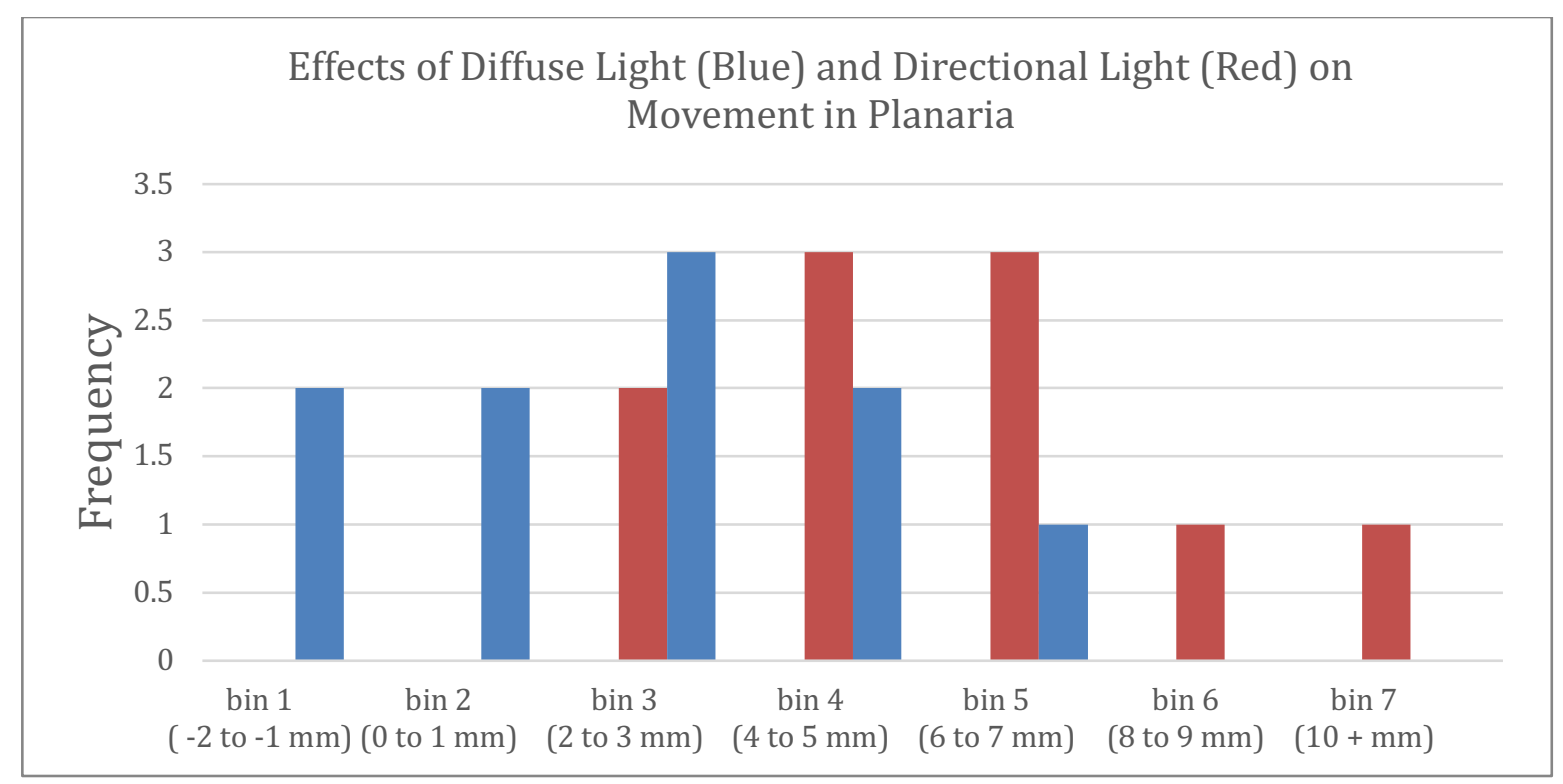

Figure 1. Histogram demonstrating distributions of movement under diffuse lighting and directional lighting. Record your observations (Question 8), construct bins (Question 9), and make a histogram (Question 10) in the Laboratory 1 Report Sheet at the end of this lab.

\section{Part E: Measures of Central Tendency}

The histogram gives you a good way to visualize the central tendency of distances traveled under each light condition. For example, the highest frequency of observations fell in bin 3 ( 2 to $3 \mathrm{~mm}$ ) for diffuse light conditions, while the highest frequencies of observations fell in bin 4 (4 to $5 \mathrm{~mm}$ ) and bin 5 (6 to $7 \mathrm{~mm}$ ) for directional light conditions.

Another common measure of central tendency that you are probably familiar with is the median. The median is the value for which half of all observations lie above, and half of all observations lie below. If we sort the observations from the reference population (diffuse light) into order from smallest to largest, we would find that the fifth observation has a value of $2 \mathrm{~mm}$, and the sixth value has a value of $3 \mathrm{~mm}$. The median then lies between 2 and $3 \mathrm{~mm}$ (we can estimate it as $2.5 \mathrm{~mm}$ ). If we sort the observations from the treatment population (directional light), we would find that the fifth observation has a value of $5 \mathrm{~mm}$, and the sixth value has a value of $6 \mathrm{~mm}$. The median then lies between 5 and $6 \mathrm{~mm}$ (we can estimate it as $5.5 \mathrm{~mm}$ ).

And of course, another obvious measure of central tendency is the mean. This is the most commonly used, although its use suggests that the data (frequency distribution) follows a normal distribution, or bell-shaped curve about the mean. If we calculate the mean distance traveled by planaria under reference conditions (diffuse light), we would get $2.0 \mathrm{~mm}$. The mean distance traveled for treatment conditions (directional light) is $5.6 \mathrm{~mm}$. When we report a mean value for a sample, it is common to also provide an estimate of the variation around this mean. Are all individual observations close to the mean, or is 
there a wide spread of observations? The standard deviation is the most commonly used measurement of the variation around that mean. It is not the purpose of this activity to teach you how to calculate standard deviation, but rather to introduce the general concept. If you do not know how to calculate standard deviation, please watch the video titled Calculating Standard Deviation (http://youtu.be/OmH3bg_v9jg). In our example, the standard deviation for the diffuse light population would be $2.67 \mathrm{~mm}$, and for the directional light population the standard deviation would be $2.55 \mathrm{~mm}$. So, we would describe our two samples as:

Reference (diffuse light) $=2.00 \pm 2.67$ (mean \pm standard deviation)

Treatment (directional light) $=5.60 \pm 2.55$

Record the median and mean values in your Lab Report sheet (Question 11) for the actual values recorded in this lab activity. For next lab, calculate the standard deviation and include this on your report sheet. You will submit this to your TA at the start of Lab 2.

\section{Part F: Assessing your Data}

Based on the data recorded in your table, you will now make a conclusion. We make the conclusion based on the null hypothesis. Remember that in this experiment, we cannot prove that light conditions increase movement of the planaria, since this may depend on other factors we have not considered. Instead, we determine if the data support the null hypothesis. If they do not, then the alternate hypothesis is more likely.

In our example:

Null Hypothesis:

"There is no difference in movement of Dugesia tigrina under diffuse and directional light conditions."

Alternate Hypothesis:

"There is a difference in movement of Dugesia tigrina under diffuse and directional light conditions."

In diffuse lighting, we saw $2.00 \pm 2.67 \mathrm{~mm}$ of movement. Under directional lighting, we saw $5.60 \pm 2.55 \mathrm{~mm}$ of movement. Based on this, it might be tempting to say planaria moved more under directional lighting than under diffuse lighting. But wait a minute! The experimental mean is used to approximate a much larger (infinite) number of planaria placed under a given lighting condition. In the diffuse lighting, the experimental mean was 2.00 , but given the variation among replicates, the true mean (of a larger, infinite number of planaria under diffuse lighting) might be as low as $-0.67 \mathrm{~mm}(2.00-$ $2.66)$, or might be as high as $4.67 \mathrm{~mm}(2.00+2.66)$. Similarly, under directional light conditions, experimental mean was 5.60 $\mathrm{mm}$, but the true mean might be as low as $3.05 \mathrm{~mm}(5.60-2.55)$ or as high as $8.15 \mathrm{~mm}(5.60+2.55)$.

Based on the mean and standard deviation, we can say that the true mean distance under diffuse lighting is likely between $-0.67 \mathrm{~mm}$ and $4.67 \mathrm{~mm}$. Under directional lighting, the true mean distance moved is likely between $3.05 \mathrm{~mm}$ and 8.15 $\mathrm{mm}$. Given this overlap in ranges, can we say there is a difference between movement under directional light conditions and diffuse light conditions? If not, we must accept the null hypothesis, there is no difference in movement of Dugesia tigrina under diffuse and directional light conditions. But how do we make this decision? We do so through the use of inferential statistics.

\section{Part G: Performing a t-test and Drawing a Conclusion}

A Student's t-test is a very common approach to for objectively comparing two samples to determine if they likely come from the same population, or if they more likely represent samples drawn from different populations. This is, perhaps, a complex way of stating t-test are used to determine if samples representing two treatment groups are statistically similar, or statistically different.

Access the video Student's t-test (https://youtu.be/fbeB9h_IIN4). View this video and calculate a t-test statistic for your data. Then draw a conclusion, based upon this test. If you calculate a value of " $t$ " that is greater than the critical value of " $\mathrm{t}$ ", then the probability that your two samples come from the same population is low (less than 5\%), and we would reject the null hypothesis, concluding that there is a difference in movement of Dugesia tigrina under diffuse and directional light conditions. If you calculate a " $t$ " value that is less than the critical value of " $t$ ", then there is greater than $5 \%$ chance that the samples represent the same statistical population, and we must accept the null hypothesis.

Calculate "t" for your data set, and also use the table (below) to determine the critical value of " $t$ ". Record these in the Laboratory Report Sheet (Question 12), along with your conclusion (i.e. do you accept or reject the null hypothesis, and is there a difference in movement by planaria under diffuse lighting versus directional lighting?) (This must be done when you come to lab 2). 


\section{Data Analysis: Laboratory Report Sheet}

(Q1 to Q4 to be completed before coming to Lab 1; Q5 to Q11 to be completed in Lab 1; Q12 to be completed before coming to $\mathrm{Lab}$ 2)

Student Name: ID \#

View the video discussion on "Scientific Method" (http://youtu.be/J8HM1i_0cIY). The video gives some examples of inductive and deductive reasoning. The video then asks you four questions, in each case you are asked to determine if an example is Inductive or Deductive. Please type either Inductive or Deductive and provide a rationale for your answer.

Question 1. Is this an example of Inductive or Deductive Reasoning?

Rationale

Question 2. Is this an example of Inductive or Deductive Reasoning?

Rationale

Question 3. Is this an example of Inductive or Deductive Reasoning?

Rationale

Question 4. Is this an example of Inductive or Deductive Reasoning?

Rationale

Experiment 1.1 Using the Scientific Method for Testing Photophobic Behavior of Dugesia tigrina

Question 5:

Asking Questions: What is your research question? Please write it below.

Question 6:

Formulating a Hypothesis: Please type your Null Hypothesis (Ho) and your Alternate hypothesis (Ha) below.

Ho:

Ha:

Question 7:

Experimental: For your experiment, please specify the Independent variable, the Dependent variable, and any controlled variable(s) below.

Independent Variable: 
Dependent Variable:

Controlled variable(s):

Question 8:

In the table below, record the distance traveled for each planarian. Reference \#1 and Treatment \#1 are your direct observations. The remaining rows will be data gathered by other students in your lab section.

\begin{tabular}{l|l|l|l|}
$\begin{array}{c}\text { Reference } \\
\text { (diffuse light) }\end{array}$ & \multicolumn{3}{c}{$\begin{array}{c}\text { Treatment } \\
\text { (directional light) }\end{array}$} \\
\hline 1 & & 1 & Distance traveled (mm) \\
\hline 2 & & 2 & \\
\hline 3 & & 3 & \\
\hline 4 & & 4 & \\
\hline 5 & & 5 & \\
\hline 6 & & 6 & \\
\hline 7 & & -3 & \\
\hline 8 & & 7 & \\
\hline 9 & & 8 & \\
\hline 10 & & 9 & \\
\hline
\end{tabular}

Question 9:

In the table below, define the distance traveled that characterizes each bin. Then record the number of observations from your table above that fall into each bin. Note that I have made allowance for 8 bins. You do not need to use all, only fill in information for the number that you consider useful for constructing a histogram.

\begin{tabular}{|c|c|c|c|c|}
\hline \multicolumn{3}{|l|}{ Bins } & \multirow[t]{2}{*}{$\begin{array}{c}\text { Reference (diffuse light) } \\
\text { \# observations }\end{array}$} & \multirow[t]{2}{*}{$\begin{array}{c}\text { Treatment (directional light) \# } \\
\text { observations }\end{array}$} \\
\hline 1( & to & $\mathrm{mm})$ & & \\
\hline 2( & to & $\mathrm{mm})$ & & \\
\hline 3( & to & $\mathrm{mm})$ & & \\
\hline 4( & to & $\mathrm{mm})$ & & \\
\hline 5( & to & $\mathrm{mm})$ & & \\
\hline 6( & to & $\mathrm{mm})$ & & \\
\hline 7( & to & $\mathrm{mm})$ & & \\
\hline 8( & to & $\mathrm{mm})$ & & \\
\hline
\end{tabular}

\section{Question 10:}

Next, use the above table to construct your histogram 


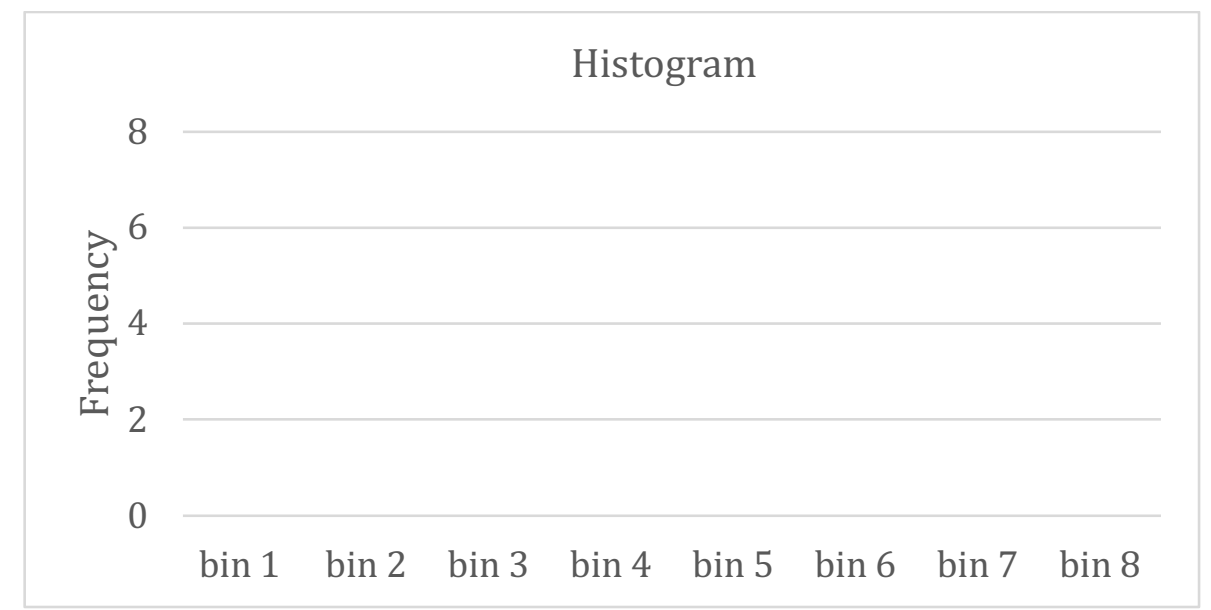

Question 11:

Record the median and mean values

$\underline{\text { Reference (diffuse light) }}$

Median $=$

Mean $=$

Standard Deviation $=$

(to be calculated before Lab 2)

$\underline{\text { Treatment (direct light) }}$

Median $=$

Mean $=$

Standard Deviation $=$

Question 12 (to be completed before Lab 2)

(to be calculated before Lab 2)

Your calculated value of " $\mathrm{t}$ " =

Critical value of " $\mathrm{t} "=$

Do you accept or reject the null hypothesis?

State your conclusion:

\section{Cited References}

Life Science Staff [Internet]. July 2012. Deductive Reasoning vs Inductive Reasoning. [July 2013] Available from: https://www.livescience.com/21569-deduction-vs-induction.html

Paskin TR, Jellies J, Bacher J, Beane WS. 2014. Planarian phototactic assay reveals differential behavioural responses based on wavelength. PLOS ONE. 9(12): e114708. 


\section{Materials}

To set up and perform this laboratory session the following items are required: brown planaria (Merlan Scientific 132954), planaria measuring devices (custom made) and LED flashlights.

To create the rectangular plastic tray (Part C: Approach) that holds the planaria, we used a plastic ruler and glued a piece of clear pipe cut longitudinally to make a trough. The ends were sealed to retain water. Using the scale provided by the ruler, the students could monitor how far, and in which direction the planaria moved.

To simulate dark conditions, we used a larger piece of black polyvinyl chloride (PVC) type pipe cut longitudinally in order to cover the trough apparatus (or an $\mathrm{L}$ shaped PVC connector). In one end of this black pipe a hole was drilled. The LED flashlight was inserted to serve as a light source to monitor planaria movement (away or closer to a light).

\section{Notes for the Instructor}

\section{Structure of the Laboratory Implementation}

We advise the following set-up for operating this introductory laboratory session: The TA will meet the laboratory coordinator/director to review the material.

The students are expected to review the lab manual and supplementary videos before the 2.5 hour session. During the in-class laboratory period, students work through the questions and participate in group exercises helping to establish a scientific community within the laboratory (e.g. Part C). The first-year biology students are expected to complete the laboratory report component prior to their next laboratory meeting. The TAs were provided with a detailed rubric during another session to serve as guidelines for evaluating the laboratory reports.

\section{Evaluation of Videos}

Overall laboratory grades from students who viewed the basic laboratory statistics video were compared to evaluate the impact of these resources in academic performance. Students who viewed the video prior to completing the laboratory report component received an average grade of $5 \%$ higher compared to students who did not view the video prior to the report. This was statistically supported using a Kruskal-Wallis test, given that the marks could not be distributed normally due to their low marking value.

We also examined the final laboratory grades, to provide insight for student performance regarding video tools. In previous offerings (2014) in the absence of supplementary tools, students obtained an average of $63 \%$. With the introduction of videos, students received an average grade of $87.3 \%$. There is no exact causation for this increase in grade, however, it is highly probable that the introduction of technology as an additional teaching tool could have contributed to the increased academic performance $(p=<0.001)$ based on the average difference. For first year biology laboratory courses, we recommend including supplementary video tools to help review the laboratory component.

\section{Cited References}

Foley AE, Herts JB, Borgonovi F, Guerriero S, Levine S C, Beilock SL. 2017. The math anxietyperformance link: A global phenomenon. Curr Dir Psychol Sci. 26(1): 52-58.

Laursen A, McCarthy H, Puddephatt KJ, de Araujo C, Victorio-Walz L. 2018. Biology 143 Laboratory Manual. Plymouth, (MI): MacMillan Learning. p. $1-17$.

Wilson SG. 2013. The Flipped Class: A Method to Address Challenges of an Undergraduate Statistics Course. Society for the Teaching of Psychology. 00(0): 17.

\section{Acknowledgments}

We are grateful to the undergraduate class of Biology I (BLG 143) 2018 for their participation. Thanks to support from the Ryerson University Learning and Teaching Grant and Ryerson University Learning and Teaching Office.

\section{About the Authors}

Charlotte de Araujo, Ph.D. is an Assistant Professor in the Department of Chemistry and Biology, Ryerson University and an educator with The G. Raymond Chang School of Continuing Education (CE).

Nicole Pereira completed her undergraduate degree in Biology during the course of this project.

Karen J. Puddephatt, M.Sc. is a laboratory technologist in the Department of Chemistry and Biology, at Ryerson University and CE Instructor.

Lynda H. McCarthy, Ph.D. is a Professor in the Department of Chemistry and Biology, Ryerson University and founder and Director of Ryerson Urban Water.

Andrew E. Laursen, Ph.D. is an Associate Professor in the Department of Chemistry and Biology, Ryerson University and Graduate Program Director for Environmental Applied Science and Management. 


\section{Mission, Review Process \& Disclaimer}

The Association for Biology Laboratory Education (ABLE) was founded in 1979 to promote information exchange among university and college educators actively concerned with teaching biology in a laboratory setting. The focus of ABLE is to improve the undergraduate biology laboratory experience by promoting the development and dissemination of interesting, innovative, and reliable laboratory exercises. For more information about ABLE, please visit http://www.ableweb.org/.

Advances in Biology Laboratory Education is the peer-reviewed publication of the conference of the Association for Biology Laboratory Education. Published articles and extended abstracts are evaluated and selected by a committee prior to presentation at the conference, peer-reviewed by participants at the conference, and edited by members of the ABLE Editorial Board. Published abstracts are evaluated and selected by a committee prior to presentation at the conference.

\section{Citing This Article}

de Araujo, C, Pereira, N, Puddephatt, KJ, McCarthy, LH, Laursen, AE. 2020. Explaining the scientific method to first year biology students in an accessible manner. Article $55 \mathrm{In}$ : McMahon K, editor. Advances in biology laboratory education. Volume 41. Publication of the 41st Conference of the Association for Biology Laboratory Education (ABLE). https://doi.org/10.37590/able.v41.art55

Compilation (C) 2020 by the Association for Biology Laboratory Education, ISBN 1-890444-17-0. All rights reserved. No part of this publication may be reproduced, stored in a retrieval system, or transmitted, in any form or by any means, electronic, mechanical, photocopying, recording, or otherwise, without the prior written permission of the copyright owner.

ABLE strongly encourages individuals to use the exercises in this volume in their teaching program. If this exercise is used solely at one's own institution with no intent for profit, it is excluded from the preceding copyright restriction, unless otherwise noted on the copyright notice of the individual chapter in this volume. Proper credit to this publication must be included in your laboratory outline for each use; a sample citation is given above. 